\title{
Integrating Brain Science Research with Intelligence Research
}

\author{
Dennis Garlick ${ }^{1}$ \\ Department of Psychology, University of Sydney, New South Wales, Australia
}

\begin{abstract}
Understanding the possible causes of differences in intelligence is crucial if children are to achieve their full potential. Such understanding has been hampered until recently, however, because researchers who study intelligence have neglected recent findings in the brain sciences suggesting that the brain develops in response to environmental stimulation. These findings have seemed to contradict intelligence research that suggests that intellectual abilities are inherited. However, the findings from intelligence research and the brain sciences can be integrated if it is accepted that there are individual differences in the process by which the brain adapts to the environment, such that some people's brains are better at adapting than others'. The findings obtained from intelligence research are consistent with this integrated model. Such an integration has implications for better understanding the nature of intelligence.
\end{abstract}

\section{Keywords}

intelligence; IQ; brain; plasticity; critical period

One of the most pressing issues facing the field of psychology is that of individual differences in intelligence (Brody, 1992; Jensen, 1998; Mackintosh, 1998). Ever since the beginning of the 20th century, when Binet first developed intelligence tests to identify school children who needed special education, researchers have recognized that identifying and understanding differences in intelligence is critical if children are to achieve their potential.

However, the field of intelligence research has been filled with controversy (Neisser et al., 1996). In 1904, Spearman reported that people who performed well at one intellectual task tended to perform well at other intellectual tasks as well. This led Spearman to suggest that there was a general factor of intelligence, or $g$, that underlay performance on these tasks. Spearman's theory generated a great debate in intelligence research about whether or not the observed positive correlation in performance across many different intellectual tasks indicates that a general factor is present, and, if it is, what the nature of this general factor might be.

The existence of positive correlations of performance across different tasks is the justification for representing intelligence by a single score, as illustrated by the Intelligence Quotient, or IQ. An IQ test does not assess all of the abilities that are important for performing successfully in all arenas of life, but many researchers argue that people who perform well on an IQ test are likely to perform well at other intellectual tasks as well. Despite the usefulness of the concept of IQ, it can lead to some confusion as to what does and does not change over time in terms of intellectual performance. For example, people's IQs are relatively stable over their lifetime, but this does not mean that their intellectual performance does not change. Rather, children's reasoning skills progressively increase through childhood, and so their performance on reasoning tests improves. A child's IQ, then, refers to his or her performance relative to other children of the same age, and the fact that IQ is stable over childhood means that a child's rank relative to other people of the same age stays the same as they get older.

It has been firmly established that intellectual performance improves during childhood, but the picture becomes more complex once maturity is reached (Cattell, 1987). Performance on some types of tasks, such as tasks assessing general knowledge and vocabulary, continues to improve throughout most of adulthood. This is not surprising, because adults can clearly still learn and acquire new knowledge. However, performance on other types of tasks, such as many traditional intelligence tests that involve reasoning, does not continue to improve after a person reaches maturity. This finding eliminates the simple explanation of intelligence that it is acquired simply through learning; if it was, intelligence (as measured by IQ tests) would continue to increase in adulthood.

Other research results are also inconsistent with a learning explanation of intelligence. For example, identical twins tend to have similar IQs, even when the twins are brought up in different environments. Moreover, adopted children tend to have IQs that are more similar to those of their biological parents than to those of their adoptive parents. These findings suggest that it is not children's upbringing but rather their genes that determines their IQ. Attempts at improving the IQ of disadvantaged children through environmental intervention programs have also been relatively unsuccessful. The typical finding is that such intervention programs have a small but significant effect on a child's IQ while the child is a part of the program. However, when the program ends, these gains are gradually lost.

In view of such findings, the general consensus in the intelligence literature is that intelligence is due to a maturational process. In other words, the development of intelligence is governed by some genetic program and is relatively immune to differences in environmental circumstances, at least within the range that most children experience.

\section{RECENT FINDINGS IN THE BRAIN SCIENCES}

Over the past 30 years, there have been considerable advances in our understanding of how the brain develops and processes information. Scientists who study the brain now believe that neural connections are critical for the meaningful processing of information (McLeod, Plunkett, \& Rolls, 1998). In other words, the pattern of activation through a network of neurons is shaped by changing the connections between the neurons. This 
suggests that different neural connections are required for different abilities. Studies of brain development have shown that these neural connections are relatively undifferentiated when a child is born and that they then become progressively more elaborate over childhood until maturity (Fig. 1).

Many studies have shown that neural connections develop and change in response to environmental stimulation (Huttenlocher, 1994; Katz \& Shatz, 1996; Kolb \& Whishaw, 1998). This capacity of the neural connections to adapt has often been referred to as neural plasticity (Nelson, 1999). For example, research has shown that if kittens are reared in a visual environment in which they are exposed only to vertical lines, in later life the only visual stimuli they will be able to recognize are vertical lines. If they are exposed only to spots of light during infancy, they will be able to see only spots of light in later life. These findings suggest that environmental stimulation plays a crucial role in determining the arrangement of a child's neural connections and, hence, how the child processes information.

Evidence that the brain adapts the neural connections to the environment seems to contradict the finding of a highly inherited general factor of intelligence. If different intellectual abilities were based on different neural connections, then they would not be expected to be related to one another, and there would not be a general factor of intelligence. In addition, if these neural connections were dependent on environmental stimulation for their development, then intelligence would be determined by a child's experiences rather than by inheritance. Because of the obvious difficulties in reconciling research findings in the two fields, researchers studying intelligence have tended to ignored the recent results of brain science research.

\section{INTEGRATING THE APPROACHES}

Intelligence researchers may have been wrong to ignore evidence that the brain adapts to the environment. Such a process would seem necessary to account for the development of the neural connections, particularly in humans, who are renowned for their ability to adapt to varying environments. However, it may not be correct to simply assume that neural plasticity is a process that is either present or absent; rather, it may be that there are individual differences in this process (Garlick, 2002). In other words, some brains may be better at adapting to the environment than others. This capacity to adapt is also likely to be inherited, as it would depend on the presence of various neural substrates that would be encoded in the genes.

Imagine a child whose brain is very good at adapting neural connections to the environment. He or she would be able to develop the appropriate connections to process many kinds of information more effectively than other children of the same age, and would appear to be bright or gifted. In contrast, consider a child whose brain is unable to adapt to the environment and who is unable to develop the neural connections necessary to understand many things experienced in the environment. That child would remain childlike as a result of poorly developed neural circuits. In short, if there are individual differences in neural plasticity, or the capacity to adapt neural connections to the environment, then some children would be able to perform better than others at many different intellectual tasks. Hence, a general factor of intelligence, or $g$, would result.

This integrated model can be demonstrated using computer simulations of neural networks in which variations in the capacity to adapt neural connections to the environment are modeled (Garlick, 2002). Such simulations have shown that a network that can adapt its connections better can learn to read faster. It can also perform better on the balance-scale task, a test in which a person must predict which side of a fulcrum will go down given different combinations of weight and distance from the fulcrum on the two sides. This task was studied by Piaget, who found that children's improved performance on the task as they got older could be described by a series of stages. Piaget attributed this improvement in performance to the process of accommodation, whereby the child's cognitive structures are modified to better reflect the environment. It has been found that a simulation of the task moves through the same stages as a child as it is given more experience with the balance scale, suggesting that the development of neural connections over childhood could explain the process of accommodation (Elman et al., 1996; McClelland \& Jenkins, 1991). A simulation comparing more and less adaptable networks showed that the more adaptable network moved through the stages faster, suggesting that it could accommodate information from the environment more effectively. Finally, a network with a faster learning rate has also been shown to perform better on the Concealed Words test, an actual intelligence test in which participants need to identify words that have been camouflaged to hide their identity. In each of these cases, varying the capacity of the network to adapt its connections has produced differences in performance that characterize differences in intelligence.

A model of intelligence that includes neural plasticity can also account for many other findings from intelligence research. For example, individual variations in neural plasticity can explain why children develop at different rates and therefore why intelligence is best represented by the concept of IQ (which measures performance of children relative to their age), rather than, for instance, performance on a test regardless of age. Such variations could also explain how intervention programs can initially assist the development of intelligence yet not have lasting effects: Improved instruction and stimulation would be expected to improve the development of even neural networks that are poor at adapting to the environment, but once the intervention ceased, the children's development would again depend on their usual level of adaptation. In comparison, children whose brains are better at adapting to the environment but who were not part of the program would then be able to slowly catch up and overtake those children who were part of the program. If there were large differences in neural plasticity and the adaptation process was relatively insensitive to environmental stimulation, then the development of intelligence could appear to be due to a maturational process, because children are largely exposed to the same environmental experiences.

If differences in intelligence were based on neural plasticity, improvements in the quality of education over time would also be expected to improve the level of intelligence over generations. In fact, performance on intelligence tests has been improving in the past century. This improvement is problematic for the argument that intelligence is determined primarily by a genetic maturational process (the traditional intelligence research stance), because the genetic makeup of the population could not have changed over such a short time. The integrated approach also has an advantage over commonly accepted views of intelligence in that individual differences in the capacity of the brain to adapt neural connections to the environment would lead to many other observed characteristics of intelligence, including the fact that more intelligent people have more complex neural 
connections, can perform better on even simple reaction time tasks, have larger brains, and show greater neural efficiency as evidenced by using less glucose in the brain when performing intellectual tasks (Garlick, 2002). In summary, individual differences in neural plasticity can account for many of the characteristics associated with differences in intelligence.

\section{IMPLICATIONS AND FUTURE DIRECTIONS}

A model of intelligence that integrates the results of intelligence research and brain science research suggests that intelligence is due to the development of neural connections in response to the environment and that differences in intelligence are due to differences in this adaptation process. This integrated approach has major implications for a number of different issues. For example, this model shows that findings in intelligence research are consistent with recent findings in the brain sciences, which means that results of brain science research can be used to better understand intelligence. Research on how the brain changes its connections in response to the environment is likely to provide information about the type of information that ultimately leads to intelligent behavior. In addition, knowing that individual differences in neural plasticity lead to differences in intelligence means that a better understanding of individual differences in intelligence is possible. Both of these advances are likely to lead to better methods of education.

The knowledge that intelligence is based on the adaptation of neural connections to the environment also suggests that there is a critical period for intellectual development (i.e., that abilities need to be learned before a certain age is reached). A critical period for intelligence seems likely because intellectual performance stops developing at maturity and is supported by research findings indicating that neural connections stop developing at this time (Bourgeois, Goldman-Rakic, \& Rakic, 2000). Thus, if people are to acquire various intellectual abilities, they need to be exposed to appropriate stimulation during childhood, while the neural circuits are still malleable. It has already been argued that there is a critical period for language (Neville \& Bavelier, 2000), and the integrated approach to intelligence suggests that other reasoning abilities are similarly affected by a critical period. This argument differs, however, from previous beliefs that early environmental experience determines a child's IQ. Rather, I am suggesting that environmental experience is important in determining whether various intellectual abilities are actually developed, given a child's level of plasticity. Another essential distinction between this model and older models is that, under the current approach, the critical period not only includes the very early years, but also extends to the years until maturity for at least some abilities. Educational practices need to take these issues into account.

There has been a lot of interest recently in finding not only the cause of differences in intelligence, but also the specific genes responsible for these differences. However, even if such genes are identified, this will not necessarily lead to an understanding of their effects. Understanding the role of these genes in the brain may facilitate identifying them and determining their precise effects.

Current imaging techniques of living brains, such as magnetic resonance imaging (MRI), are likely to be of less help in determining the cause of differences in intelligence. Such differences are likely to be at the level of the specific neural connections, and current imaging techniques would need to increase their resolution by 1,000 times in order to show this level of detail. Mapping neural connections is likely to be even more problematic than identifying their complexity because of the sheer number of connections involved and the challenges inherent in precisely tracing the connections and determining their function. For example, the neurons shown in Figure 1 were stained using a method that results in only a few neurons in each slice being stained. If all of the neurons in each of the slices had been stained, then the illustrations would be completely black. Researchers hope that improvements in tracing techniques will allow a more detailed picture of neural connections to be constructed in the future.

In summary, by integrating results of brain science research with those of intelligence research, a better understanding of intelligence can be achieved. The recent findings in the brain sciences suggest that intelligence is not due simply to an inherited maturational process, but rather arises from the development of neural connections during childhood in response to environmental stimulation. Such a reconceptualization has major implications for education as well as a number of other issues in the field.

\section{Recommended Reading}

Bourgeois, J.-P., Goldman-Rakic, P.S., \& Rakic, P. (2000). (See References)

Elman, J.L., Bates, E.A., Johnson, M.H., Karmiloff-Smith, A., Parisi, D., \& Plunkett, K. (1996). (See References)

Garlick, D. (2002). (See References)

Kolb, B., \& Whishaw, I.Q. (1998). (See References)

Neisser, U., Boodoo, G., Bouchard, T.J., Jr., Boykin, A.W., Brody, N., Ceci, S.J., Halpern, D.F., Loehlin, J.C., Perloff, R., Sternberg, R.J., \& Urbina, S. (1996). (See References)

\section{Note}

1. Address correspondence to Dennis Garlick, Department of Psychology, University of Sydney, New South Wales, 2006, Australia.

\section{References}

Bourgeois, J.-P., Goldman-Rakic, P.S., \& Rakic, P. (2000). Formation, elimination, and stabilization of synapses in the primate cerebral cortex. In M.S. Gazzaniga (Ed.), The new cognitive neurosciences (2nd ed., pp. 45-53). Cambridge, MA: MIT Press.

Brody, N. (1992). Intelligence (2nd ed.). San Diego, CA: Academic Press.

Cattell, R.B. (1987). Intelligence: Its structure, growth and action. Amsterdam: North Holland.

Elman, J.L., Bates, E.A., Johnson, M.H., Karmiloff-Smith, A., Parisi, D., \& Plunkett, K. (1996). Rethinking innateness: A connectionist perspective on development. Cambridge, MA: MIT Press.

Garlick, D. (2002). Understanding the nature of the general factor of intelligence: The role of individual differences in neural plasticity as an 
explanatory mechanism. Psychological Review, 109, 116-136.

Huttenlocher, P.R. (1994). Synaptogenesis, synapse elimination, and neural plasticity in human cerebral cortex. In C.A. Nelson (Ed.), Threats to optimal development: Integrating biological, psychological, and social risk factors (pp. 35-54). Hillsdale, NJ: Erlbaum.

Jensen, A.R. (1998). The g factor. Westport, CT: Praeger.

Katz, L.C., \& Shatz, C.J. (1996). Synaptic activity and the construction of cortical circuits. Science, 274, 1133-1138.

Kolb, B., \& Whishaw, I.Q. (1998). Brain plasticity and behavior. Annual Review of Psychology, 49, 43-64.

Mackintosh, N.J. (1998). IQ and human intelligence. Oxford, England: Oxford University Press.

McClelland, J.L., \& Jenkins, E. (1991). Nature, nurture, and connections: Implications of connectionist models for cognitive development. In K. VanLehn (Ed.), Architectures for intelligence: The twenty-second Carnegie Mellon symposium on cognition (pp. 41-73). Hillsdale, NJ: Erlbaum.

McLeod, P., Plunkett, K., \& Rolls, E.T. (1998). Introduction to connectionist modelling of cognitive processes. Oxford, England: Oxford University Press.

Neisser, U., Boodoo, G., Bouchard, T.J., Jr., Boykin, A.W., Brody, N., Ceci, S.J., Halpern, D.F., Loehlin, J.C., Perloff, R., Sternberg, R.J., \& Urbina, S. (1996). Intelligence: Knowns and unknowns. American Psychologist, 51, 77-101.

Nelson, C.A. (1999). Neural plasticity and human development. Current Directions in Psychological Science, 8, 42-45.

Neville, H.J., \& Bavelier, D. (2000). Specificity and plasticity in neurocognitive development in humans. In M.S. Gazzaniga (Ed.), The new cognitive neurosciences (2nd ed., pp. 83-98). Cambridge, MA: MIT Press.

Poliakov, G.I. (1959). Progressive neuron differentiation of the human cerebral cortex in ontogenesis. In S.A. Sarkisov \& S.N. Preobrazenskaya (Eds.), Development of the central nervous system (pp. 11-26). Moscow: Medgiz. 